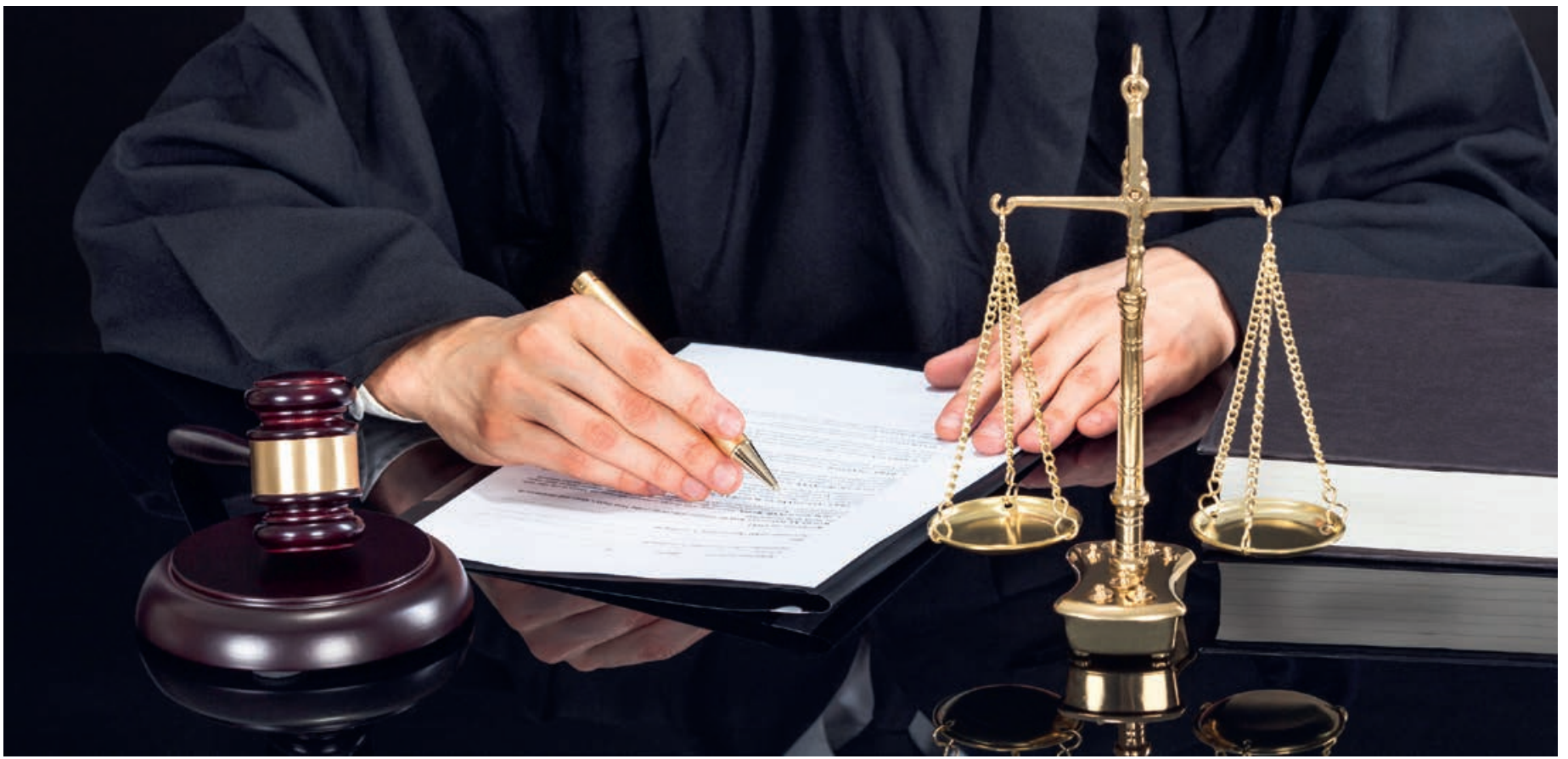

\title{
Halbgötter in Schwarz
}

\section{Josef E. Brandenberg}

Dr. med., Präsident der FMCH

\section{Résumé}

Depuis des années, notre médecine est régie par les demi-dieux en noir. Le traitement curatif est devenu une infraction pénale, à moins que le patient n'accepte la «blessure physique». Les tribunaux administratifs et les cours des assurances sociales rendent eux aussi régulièrement des décisions dans des affaires médicales. Le Tribunal fédéral a ainsi récemment statué que «tout être humain doit être présumé en bonne santé». Le 14 septembre 2018, le Tribunal administratif fédéral a défini la qualité des chirurgiens de la manière suivante: "l'exercice fait le maître.» Fixer un nombre minimum de cas dans le canton de Zurich est de ce fait justifié, même si les motifs relèvent plus de la planification et de la rentabilité que de la qualité. Dans son arrêt du 29 mars 2018 sur l'intervention tarifaire du Conseil fédéral en 2014, le Tribunal fédéral a déclaré que le Conseil fédéral peut également tenir compte d'enjeux politiques lors de I'ajustement du tarif. II contrevient ainsi au principe de l'adéquation d'un tarif et se fait le complice de la sphère politique.

Il est inutile de critiquer les tribunaux, parce que les demi-dieux en noir ont toujours raison. Evitons autant que possible de saisir la justice, parce qu'on ne peut pas négocier avec les tribunaux. Le monde juridique des normes est inconciliable avec le monde médical des probabilités et des expériences. Soyons de bons médecins, documentons la qualité et parlons aux patientes et patients.

Wie auf Nadeln sass ich im ersten Zug von Luzern Richtung Genf. Bei den letzten beiden Bahnfahrten in die Westschweiz strandete ich wegen Betriebsstörungen einmal in Romont, einmal in Fribourg. Jetzt durfte das nicht passieren. Unter Strafandrohung musste ich um 9.15 Uhr vor dem Polizeigericht in Nyon als Zeuge erscheinen. Einen späteren Termin gestattet man nicht. In der Eingangshalle huschten Gestalten in schwarzen Talaren mit Seidenrevers, weiten Ärmeln, teilweise mit Pelz bestückten Säumen vorbei. Vom Podium herab befragte der Richter in schwarzer Robe den Zeugen. Aus dem Off stellten die Anwälte - auch sie in Schwarzihre Fragen. Zentrales Thema: Patientenaufklärung. Auf die Frage des Vorsitzenden, warum die Ärztinnen und Ärzte ein schriftliches Aufklärungsprotokoll verfassten, antwortete ich "weil es die Richter so wollen". Nach einer Stunde war das Kreuzverhör vorbei, das Protokoll unterschrieben, der Zeuge ohne Entschädigung und spesenfrei entlassen.

In der Tat verändern seit Jahren die Halbgötter in Schwarz unsere Medizin. Die Heilbehandlung ist zur Straftat geworden - ausser der Patient stimmt der «Körperverletzung» zu. Jeder Patient ein Masochist? Zwar nicht in schwarzen Talaren und fast ausschliess- 
lich nach Aktenlage urteilen auch die Gerichte im Bereich der Sozialversicherungen immer wieder über medizinische Belange. Das Bundesgericht beschränkte sich im Urteil vom 21. März 2018 nicht auf die Beurteilung von IV-Leistungen [1], sondern entschied, jeder Mensch sei primär als gesund zu betrachten [2].

\section{Die Heilbehandlung ist zur Straftat geworden -} ausser der Patient stimmt der «Körperverletzung» $\mathrm{zu}$.

Auch Verwaltungsgerichte halten sich nicht zurück und machen zunehmend Medizin, wie das Urteil vom 14. September 2018 dokumentiert [3]. Beim Begriff "Qualität» handle es sich um eine "offene und unbestimmte Umschreibung einer Anforderung an einen Leistungsauftrag, die einer wertenden Konkretisierung bedürfe. Der unbestimmte Rechtsbegriff sei daher der Auslegung durch das Gericht zugänglich.» Mit anderen Worten: Das Gericht sieht sich befugt und berufen, die medizinische Qualität zu definieren. Hintergrund ist eine Beschwerde eines Zürcher Spitals beim Bundesverwaltungsgericht gegen die Einführung von Mindestfallzahlen für Operateure [4]. Und wie definieren die Richter die medizinische Qualität? Es sei «grundsätzlich unumstritten, dass hoch qualifizierte Tätigkeiten, wie die infrage stehenden chirurgischen Eingriffe, ein hohes Mass an kontinuierlicher Übung verlangen". Der allgemein bekannte Grundsatz «Übung macht den Meister» gelte hier ganz besonders, da sich "operative Fehler auf die betroffenen Patientinnen und Patienten tödlich auswirken können». Dieser simplen Plattitüde kann man nur mit einem anderen Sprichwort entgegnen: «Schuster bleib bei deinem Leisten!» Immerhin wird bestätigt: Operieren ist eine hochqualifizierte und gefährliche Tätigkeit. Die Beibehaltung der quantitativen Dignitäten im Tarif wäre damit höchstrichterlich begründet. Aber eigentlich geht es dem Gericht ebenso wenig um Qualität wie der Zürcher Gesundheitsbehörde [5]. Auf den 65 Seiten des Urteils kommt der Begriff 145 Mal vor, 32 Mal in Verbindung mit «Wirtschaftlichkeit, Preis und Effizienz» und 94 Mal mit «Planung, Kontrolle, Anforderung, Nachweis». Es geht um Planung und Wirtschaftlichkeit, um Planwirtschaft.

Wie die Gerichte die Übergriffe der Politik auf die Medizin stützen und schützen, zeigt auch das jüngste Urteil aus dem Kanton Aargau [6]. Das Verwaltungsgericht setzte die kantonale Liste «ambulant vor stationär» ausser Kraft. Das Urteil wurde ans Bundesgericht weitergezogen. Wie dieses auch immer entscheiden wird, nicht die Rechtmässigkeit der Liste an sich steht zur Diskussion, sondern einzig die Zuständigkeit, ob das BAG oder die Kantonsbehörden über die medizinische Indikation für einen stationären Aufenthalt zu entscheiden befugt sind.

Mit dem Urteil vom 29. März 2018 ist das Bundesgericht definitiv zum Erfüllungsgehilfen der Politik geworden [7]. Es bejaht die Rechtmässigkeit des Tarifeingriffs von 2014 in der Medienmitteilung vom 13. April 2018 und begründet seine Missachtung der gesetzlich vorgesehenen Sachgerechtigkeit eines Tarifs wie folgt: «Der Bundesrat darf bei der Anpassung von Taxpunkten der TARMED-Tarifstruktur lineare Kürzungen bei verschiedenen Positionen vornehmen und dabei auch politischen Anliegen Rechnung tragen» [8].

Kollege Urbaniok fragte kürzlich, ob man Gerichte kritisieren dürfe [9]. Die Frage erübrigt sich. Besser fragt man: Was bringt Kritik an Gerichtsurteilen? Antwort: nichts. Denn mit Gerichten kann man nicht diskutieren und schon gar nicht verhandeln. Die Halbgötter in Schwarz haben immer Recht. Und kommt es zum Rekurs, urteilen die Halbgötter der letzten Instanz abschliessend $[3,7]$.

Hören wir auf, Gerichte anzurufen. Sparen wir uns die vielen Rechtsgutachten. Die juristische Welt der Normen steht in unvereinbarem Gegensatz zur medizinischen Welt der Wahrscheinlichkeiten und Erfahrungen. Verhandeln wir, wo wir verhandeln können. Festigen wir die Einigkeit innerhalb der Ärzteschaft.

Die juristische Welt der Normen steht in unvereinbarem Gegensatz zur medizinischen Welt der Wahrscheinlichkeiten und Erfahrungen.

Vor allem aber: Machen wir gute Medizin, dokumentieren wir die Qualität und - ganz entscheidend - reden wir mit den Patientinnen und Patienten. Kurz: Besinnen wir uns wieder auf die Stärken des «weissen Kittels», ohne in den überheblichen Rausch zu verfallen, Halbgötter zu sein.

\section{Bildnachweis}

(c) Andrey Popov | Dreamstime.com

\section{Literatur}

1 BGE 144 V 50 vom 21.3.2018.

2 Jeger J. «Der Mensch ist gesund». Jusletter 8. Oktober 2018.

3 Urteil C-5603/2017 BVerGer, Abteilung III vom 14.9.2018.

4 Beschwerde der Spital Bülach AG vom 29. September 2017 ans Bundesverwaltungsgericht.

5 Brandenberg JE. Mindestfallzahlen - Qualität oder Surrogat? Schweiz Ärzteztg. 2018;99(47):1647-8.

6 Aargauer Zeitung vom 13.12.2018

7 BGE 144 V 138 vom 29.3.2018.

8 Medienmitteilung des Bundesgerichtes vom 13.4.2018

9 F. Urbaniok. Darf man das? Psychiater Frank Urbaniok reagiert auf die Empörung über seine Gutachten-Kritik. Schweiz am Wochenende. 22.12.2018. 